\title{
Air void analysis of hardened concrete by means of photogrammetry
}

Wolter, Sofie; Uhre, Frederik Alexander Hvelplund; Hasholt, Marianne Tange; Dahl, Vedrana Andersen; Anton, François

\section{Published in:}

Construction and Building Materials

Link to article, DOI:

10.1016/j.conbuildmat.2019.07.203

Publication date:

2019

Document Version

Peer reviewed version

Link back to DTU Orbit

Citation (APA):

Wolter, S., Uhre, F. A. H., Hasholt, M. T., Dahl, V. A., \& Anton, F. (2019). Air void analysis of hardened concrete by means of photogrammetry. Construction and Building Materials, 226, 953-964.

https://doi.org/10.1016/j.conbuildmat.2019.07.203

\section{General rights}

Copyright and moral rights for the publications made accessible in the public portal are retained by the authors and/or other copyright owners and it is a condition of accessing publications that users recognise and abide by the legal requirements associated with these rights.

- Users may download and print one copy of any publication from the public portal for the purpose of private study or research.

- You may not further distribute the material or use it for any profit-making activity or commercial gain

- You may freely distribute the URL identifying the publication in the public portal 
This is a preprint of an article published in Construction and Building Materials:

Sofie Wolter, Frederik Alexander Hvelplund Uhre, Marianne Tange Hasholt, Vedrana Andersen Dahl, François Anton:

"Air void analysis of hardened concrete by means of photogrammetry"

Construction and Building Materials 226 (2019) 953-964

The published version is available online:

https://doi.org/10.1016/i.conbuildmat.2019.07.203 


\title{
Air void analysis of hardened concrete by means of photogrammetry
}

\author{
Sofie Wolter, Frederik Alexander Hvelplund Uhre, Marianne Tange Hasholt \\ Technical University of Denmark, Brovej, Building 118, room 066, 2800 Kgs. Lyngby \\ Vedrana Andersen Dahl, Francois Anton
}

\begin{abstract}
The present study investigates the use of close-range photogrammetry for automated air void analysis of hardened concrete without contrast enhancement.

First, polished plane sections of concrete with varying air contents were analyzed by photogrammetry. The data in the form of point clouds were processed to identify the air voids. Second, the same plane sections were subjected to traditional air void analysis by contrast enhancement. Results of both methods were compared.

The method based on photogrammetry showed potential, but it could not capture the smallest air voids. Better camera resolution and/or methods to reduce noise in the point cloud are needed.
\end{abstract}

Keywords: Concrete, Air void analysis, Photogrammetry, Image analysis

\section{Introduction}

The presence of air voids is beneficial for concrete frost resistance. This was discovered in the 1930es [1], and the decade after, Powers proposed to use the spacing factor as measure when evaluating the protection provided by a certain air void system

5 [2]. He also demonstrated how to determine the spacing factor from linear traverse analysis of a concrete plane section. The method proposed by Powers is in principle

Email addresses: s143886@student.dtu.dk (Sofie Wolter), s133930@student.dtu.dk (Frederik Alexander Hvelplund Uhre), matah@byg.dtu.dk (Marianne Tange Hasholt)

Preprint submitted to Construction and Building Materials

June 3, 2019 
identical to the method described in ASTM C 457 (procedure A) [3], which has been in force since the 1960es [4].

According to the reference method of ASTM C 457, it is a human operator, who determines which parts of the traverse line are within air voids and which parts are not. However, the employment of a human operator may have some disadvantages. The main disadvantage is that when the test outcome is based on judgements made by an operator, all operators may not reach the exact same result; the test becomes operator dependent [5, 6, 7, 8]. In addition, it may be difficult to find skilled personnel, and a heavily labor demanding test method makes it expensive to carry out the test.

The disadvantages are also motivations for replacing the human operator by machine vision or automated image analysis. This has indeed already been done, see e.g. [4, 9, 10]. In these methods, it is custom to apply some kind of contrast enhancement of the plane section to make it easier for the computer to distinguish the air voids. ${ }_{20}$ Typically, the plane section is first painted dark or black all over, and then the air voids are filled with a white substance that makes the air voids stand out from the dark background. The contrast enhancement is e.g. described in the European standard EN 480-11 [11], and the 2016 version of ASTM C 457 also includes a procedure for contrast enhancement [3].

However, the contrast enhancement may be problematic, too. Below are some of the pitfalls listed:

a. The dark ink may fill up the air voids, so they later are inaccessible for the white substance.

b. The white substance can be a white powder, e.g. barium sulfate $\left(\mathrm{BaSO}_{4}\right)$ or a white paste, e.g. a mixture of zinc oxide ( $\mathrm{ZnO})$ and vaseline. In either case, the grain size of the powder is decisive for the minimum air void size that can be detected.

c. The distinction between black and white is typically made by defining a threshold value, as the image of the surface contains a range of grey tones. If the threshold value is subjectively defined by an operator, the test is still operator dependent. 
d. The white substance used to highlight the air voids will be present in all indents and grooves of the plane section surface - not only air voids but also e.g. pull-outs accidentally made during specimen preparation, porosities in aggregates [12, 13], and hollow fly ash particles [13, 14]. The operator will know the difference, but the computer will interpret all white areas of the plane section as air voids. Pleau et al. compared results from image analysis and visual examination by a human operator [15]. They found that for air voids in the size interval $0-80 \mu \mathrm{m}$, the number of air voids identified by the image analysis test system is $50-70 \%$ higher than number of air voids identified by the human operator. Even though the test standard may require that the test surface is inspected and local mis-colored areas are re-touched with a felt-tipped pen, it is only the largest defects that are visible by the naked eye, whereas most of the defects are small and will pass the inspection.

The objective of the present project was to identify an automated procedure that could be carried out without the contrast enhancement.

In the contrast enhancement procedure, the white substance can be pressed into the air voids, because the air voids are hollows relative to the plane section surface. Like this, identifying air voids is a parallel problem to identifying hollows. In geodesy, the classical approach to identifying hollows in a landscape is photogrammetry [16]. In photogrammetry, the location of a point in $3 \mathrm{D}$ is determined by observing the same point in multiple photos. For this reason, it was decided to test whether close range photogrammetry can be used to identify the air voids on a plane section. If this is possible, it immediately solves points a-c in the list above. It does not solve that some hollows are not air voids, but this may be possible to solve by image analysis at a later point. For example, Song et al. [17] have shown that it is possible to identify porosities in aggregates, when paste and aggregates at the plane surface of the test specimen are not covered by ink. If it is possible to perform air void analysis by using photogrammetry, it may also open new possibilities for performing air void analysis with inexpensive equipment, as many cell phones today have built-in digital cameras of relatively high 65 resolution. 


\section{Theory}

\subsection{Air void parameters}

Research shows that both the amount of air and the size distribution of the air voids are of great importance for concrete frost resistance [2]. However, if the concrete holds to Mehta and Monteiro [18], an increase of 5\% air will reduce the strength by $25 \%$.

Therefore it is important to be able to quantify the air void structure to make concrete that is both strong and frost resistant, so the frost resistance of the concrete can be assessed.

\subsubsection{Specific surface}

The specific surface, $\alpha$, describes the ratio between the surface area and the volume of the air voids, and can be derived from linear travers analysis of the concrete. The specific surface can be calculated by [2]:

$$
\alpha=4 / \bar{l}=4 N / T_{a}
$$

where $\mathrm{N}$ is the number of air voids intersected, $T_{a}$ is the traverse length through air voids and $\bar{l}$ is the average chord length though air voids.

\subsubsection{Spacing factor}

The spacing factor is related to the biggest possible distance to the nearest air void. To calculate the spacing factor some assumptions are made.

The spacing factor is calculated from the ratio between the cement paste content and the measured air content along a traverse line in the concrete sample [2], given by:

$$
p / A=T_{p} / T_{a}
$$

where $\mathrm{p}$ is the cement paste content, $\mathrm{A}$ is the air content, $T_{p}$ is the traverse length through cement paste, and $T_{a}$ is the traverse length through air voids. If $p / A \leq 4.342$ (i.e. the air content is high) the spacing factor is derived from the traverse line through

${ }_{85}$ the cement paste and the number of intersected air voids. If $p / A>4.342$ it is assumed 
that the air voids are uniformly distributed, and if a cube is formed in the concrete where every corner represents an air void, the spacing factor will be equal to half the length of the diagonal [2].

From the relation $p / A$, the spacing factor, $\bar{L}$, can be calculated by:

$$
\bar{L}= \begin{cases}T_{p} / 4 N, & \text { if } p / A \leq 4.342 \\ 3 / \alpha\left[1.4(1+p / A)^{1 / 3}-1\right], & \text { if } p / A>4.342\end{cases}
$$

where $\mathrm{N}$ is the number of air voids intersected, and $\alpha$ is the specific surface [2].

A high number of uniformly distributed and small air voids will result in a small spacing factor and a large specific surface. In order to optimise the frost resistance of hardened concrete, without compromising the strength, a small spacing factor and a large specific surface is therefore needed.

\subsection{Photogrammetry}

Photgrammetry is a method of making measurements by analysing photographs. It is used in different fields such as mapping and land surveying, but also in the investigation of objects at close range. A photograph is captured via a perspective projection, meaning that the coordinates defining the object's geometry are projected through a fixed point called the perspective centre [19].

\subsubsection{Basic photogrammetry}

In a perspective projection a 3-dimensional object is projected into the 2-dimensional image plane [19]. The relation between the size of the object in the image plane and the size of the real object is significant in photogrammetry, as it describes the quality of the projection.

The relation between the length of the object and the corresponding length in the image plane equals the relation between the distance from the image plane to the perspective centre (the principal distance), and the distance between the perspective 
center and the object, see figure 1 The geometric relation is given by (4):

$$
d_{i} / d_{o}=c / h
$$

where $d_{o}$ is the length in the object plane, $d_{i}$ is the corresponding length in the image plane [19], $c$ is the distance between the image plane and the perspective centre and $\mathrm{h}$ is the distance between the perspective centre and the object.

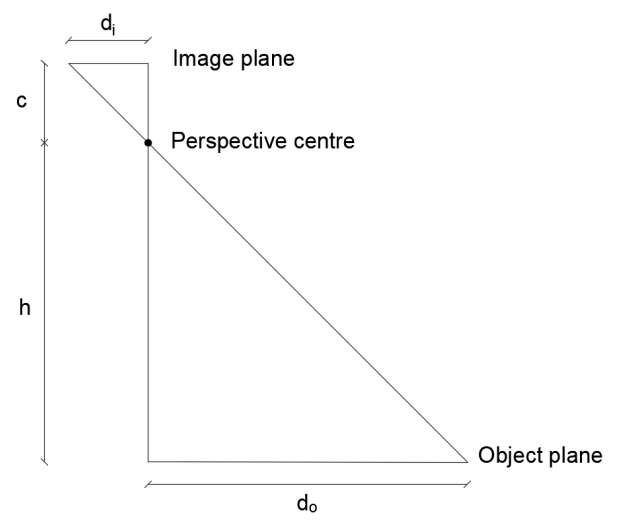

Figure 1: Principle sketch of geometrical relations

\subsubsection{Specifications for camera and objective}

By knowing the specifications for the camera and the objective used, several of the parameters from equation (4) can be derived. The focal length, $f$, is an objective determined constant, which describes the distance between the image plane and the perspective centre, with infinity focus [20]. When analysing pictures taken from great distances, the focal length can be used as an approximation of the actual distance $c$ [20]. When shooting close-range photographs, the following will apply [19]:

$$
c>f
$$

\subsubsection{Digital photogrammetry}

A digital photograph is an array consisting of a high number of rectangular or quadratic elements [19]. These elements are called pixels, meaning picture elements. 
Each pixel holds a numerical representation of a brightness or a colour. The resolution of a camera describes the total number of pixels contained by the photographs, produced by the camera, and it is typically measured in megapixels. The geometry of pixels is described by the pixel size. The focus distance $f_{d}$, is an objective specification, which defines the minimal distance between the objective and the object, where the camera is in focus.

When taking photographs at close range, the smallest size of a projectable object is of interest. This size is defined in this article as the minimum object size, MOS, and it depends on the pixel size, $s_{\text {pixel }}$, the principle distance, as well as the distance from the camera to the object. The relation is derived from equation (4) and is shown on figure 2]as:

$$
s_{\text {pixel }} / \operatorname{MOS}=c / h \Leftrightarrow M O S=\left(s_{\text {pixel }} \cdot h\right) / c
$$

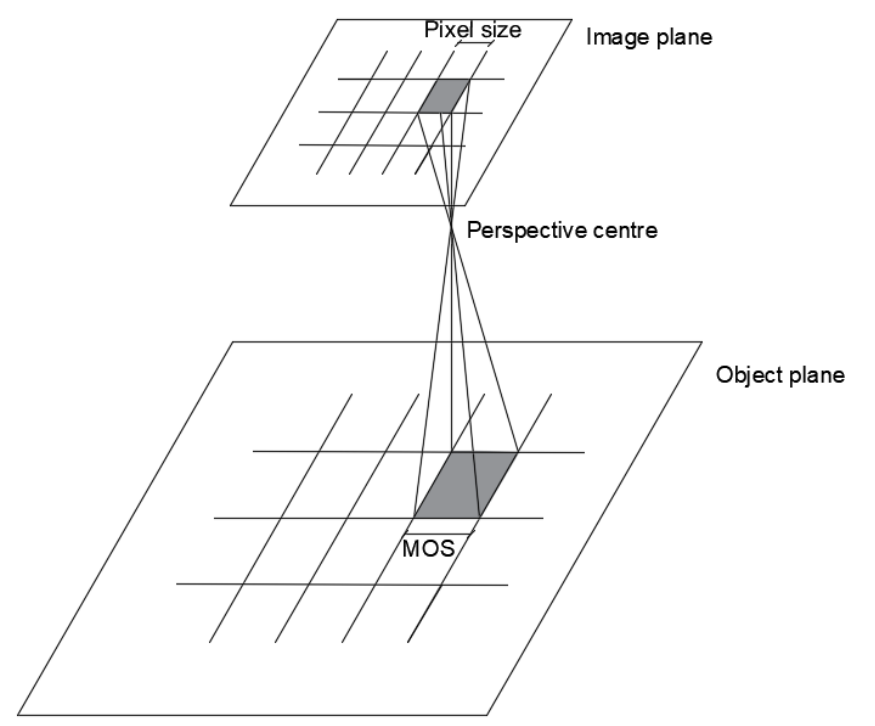

Figure 2: Principle sketch of the influence of the pixel size [19] 


\subsubsection{Photogrammetry in practice}

The collection of photographs can be digitally processed to obtain 3D positions of the terrain resulting in a digital elevation model (DEM), see Section 2.2.5. The overlap between the pictures must be at least $60 \%$ of the area of each picture to obtain results of good quality [19].

140 When analysing objects at close range, a similar procedure as used in land surveying can be used. When appropriate, a series of photographs of the object may be taken from different angles. This is the setting used for analysing the concrete specimens.

The principle distance, $c$, is an unknown theoretical value. As equation (6) is valid, it can be derived that by using the focal length, $f$, instead of the principle distance in equation (2), the calculated value of the MOS will increase. It is therefore conservative to use the focal length in the calculation, and equation (6) can be rewritten as:

$$
M O S=\left(s_{\text {pixel }} \cdot h\right) / f
$$

\subsubsection{Point clouds}

When the same point is observed in photographs taken from different viewpoints, the 3D coordinates of the point can be determined as an intersection of lines, where each photograph contributes with one line in 3D. This requires the camera pose and the projection parameters of the camera to be known. Camera pose and projection parameters can be estimated simultaneously with the 3D coordinates of a set of points through the procedure called bundle adjustment. Bundle adjustment attempts to minimize the discrepancy between the observed and the predicted projections of the set of 3D points to the sequence of photographs. Depending on an application, the projection parameters of the camera may be estimated in the pre-processing step (camera calibration). The obtained points are defined in terms of their $\mathrm{x}, \mathrm{y}$ and $\mathrm{z}$ coordinates. Initially, there is no relation between the points and such a collection of points is referred to as a point cloud. Since the obtained point cloud approximates the surface of the object, it is of interest to use the points for creating a surface model. 


\subsubsection{Delauney triangulation}

When processing the point cloud data, triangulation is often used, where the points are connected in triangles. The triangles create a surface that approximates the surface of the object. One of the most commonly used forms of triangulation is the Delauney triangulations. In 2D, the Delauney triangulation is generated from the following principle:

Two nearby points are analysed at the same time. If it is possible to generate a circle which passes through both points, without any other points laying inside the interior of the circle, then an edge connecting the two points is generated. This is called a Delauney edge. A Delauney triangle is a triangle consisting of 3 vertices connected by Delauney edges. Furthermore, it must be possible to generate a circle passing through all three points, without any other point lying within the circle. The Delauney triangles form a Delauney triangulation. A triangulation is defined as a Delauney triangulation, 175 if all possible Delauney edges are a part of the triangulation.

An approach for generating a triangulation for 3D point cloud using 2D Delauney triangulation involves only considering $\mathrm{x}$ and $\mathrm{y}$ coordinate of a point when connecting the points in the triangles. This will yield a satisfactory result if points lie close to a horizontal plane, but the quality of triangulation may decrease if the $\mathrm{z}$ coordinates of the points vary.

\section{Materials and methods}

\subsection{Test specimens}

Five concrete mixes with different air contents were cast. The five mixes all had a w/c ratio of 0.45 and a desired paste content of $36.8 \%$. The air content was adjusted using an air entraining agent. Table 1 shows the mix design for the reference mix without air entrainment.

A total of 35 liter concrete was cast. Before mixing, the moisture content of the aggregates was measured, and the amount of mixing water was adjusted accordingly. The air entraining agent used was an an-ionic surfactant based on sodium (C14-C16) 
Table 1: Mix design for $1 \mathrm{~m}^{3}$, aggregates in SSD-state

\begin{tabular}{ll}
\hline Material & Amount $[\mathbf{k g}]$ \\
\hline Cement & 478.9 \\
Water & 215.5 \\
Sand, size 0-4 mm & 831.0 \\
Granite, size 4-8 mm & 166.2 \\
Granite, size 8-11 mm & 664.8 \\
\hline
\end{tabular}

olefin sulfonate. According to the technical data sheet, the recommended dosage is $0.5 \%$, relative to the cement mass. Concrete mixes were prepared with dosages of $0.0 \%, 0.05 \%, 0.1 \%, 0.2 \%$, and $0.5 \%$ to obtain different air void structures for all 5 specimens. The air content of the fresh concrete was measured by pressuremeter. For each mix, $1 \varnothing 150 \times 300 \mathrm{~mm}$ cylinder was cast.

1951 day after casting the concrete specimens, they were taken out of the moulds and placed in a water bath. After 7 days, the concrete was placed in a climate chamber $\left(20 \pm 2^{\circ} \mathrm{C}\right.$ and $65 \% \mathrm{RH}$ ) until preparation for air void analysis.

\subsection{Traditional air void analysis}

The air void analysis of the hardened concrete was made in compliance with the American standard ASTM C 457 [3].

The air void analysis of the hardened concrete was made in compliance with the American standard ASTM C 457 [3]. Each of the 5 concrete specimens were cut from the center of the concrete cylinder and had the dimensions 100x105x20 mm. Each ${ }_{205}$ specimen was ground with abrasive grains in 3 steps with grain size 240,600 , and 1000, respectively. After the contrast enhancement, the specimens were examined with an automated ASTM C-457 analyzer, performing linear transverse analysis. The equipment was developed by DBT (Dansk Beton Teknik). It has a resolution of 2 micron, it is fully documented and has been applied for ILAC accredited air void analysis for many years. 


\subsection{New method based on photogrammetry}

The new air void analysis required the use of photogrammetry, in order to generate point clouds, which would approximate the geometry of the actual concrete surfaces. Pictures of the specimens were taken, using a camera and objective with specifications and setup listed in section 3.3.1 and 3.3.2 Pointclouds were generated from the pictures using software as described in section 3.3 .3 The pointclouds were then analysed as described in section 4

\subsubsection{Camera equipment}

The camera used in this project was a Nikon DS610, with an "AF-S VR MICRONIKKOR 105MM F/2.8G IF-ED" objective. Specifications for the camera and the objective is listed in table 2

Table 2: Technical specifications for Nikon D610 and AF-S VR MICRO-NIKKOR 105MM F/2.8G IF-ED objective

\begin{tabular}{|c|c|c|}
\hline & Technical specifications & Value \\
\hline \multirow{5}{*}{ 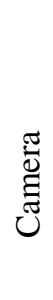 } & Resolution & 24.3 Megapixel \\
\hline & Picture censor & $35.9 \times 24.0 \mathrm{~mm}$ \\
\hline & Pixel size & $5.97 \mu \mathrm{m}$ \\
\hline & Focal length & $105 \mathrm{~mm}$ \\
\hline & Smallest focus distance & $314 \mathrm{~mm}$ \\
\hline \multirow{3}{*}{$\begin{array}{l}\stackrel{0}{0} \\
: 0 \\
0 \\
0\end{array}$} & Aperture & $\mathrm{f} / 2.8$ \\
\hline & Shutter time & $1 / 160$ second \\
\hline & ISO & 2000 \\
\hline
\end{tabular}

From equation (7) the size of the smallest projectable object can be derived:

$$
\operatorname{MOS}=(5.97 \mu \mathrm{m} \cdot 314 \mathrm{~mm}) / 105 \mathrm{~mm}=17.85 \mu \mathrm{m}
$$




\subsubsection{Set-up and photo shooting}

The specimens were photographed after each step of the grinding process, before shifting to a finer grain size, i.e. three sets of photos are obtained for each concrete mix corresponding to the three levels of grinding.

Ideally, the new method should work using any realistic lightning conditions. For this initial study the lightning conditions were controlled. This was done using two spotlights facing towards the ceiling as well as normal ceiling light, see Figure 3 .

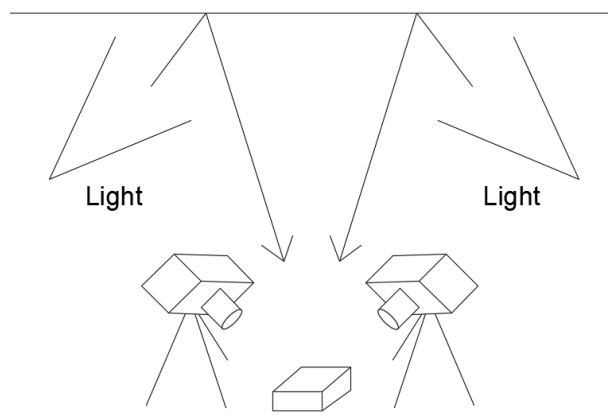

Figure 3: Set-up of camera and lamps for the photo shooting

The specimens were placed on a white surface between the two spotlights. The tripod with the camera was then placed near the specimen.

The specimens were photographed from four different angles, corresponding to the four corners of the sample. The images were taken from three different distances from each angle, resulting in total of 12 images.

Every corner of the specimens were photographed from three different distances. The smallest focus distance for the objective was $314 \mathrm{~mm}$, see table 2, so the pictures could not be taken closer to the concrete than this. In order to make sure the pictures were in focus, the smallest distance to the specimens was set to $350 \mathrm{~mm}$.

The overlap of the photos should be at least $60 \%$, see section 2.2.4 In order to find the distance with $60 \%$ overlap, the focus distance was divided by $\sqrt{0.6}$ :

$$
350 \mathrm{~mm} / \sqrt{0.6}=452 \mathrm{~mm}
$$

${ }_{235}$ Thus the next four pictures were taken with distance $450 \mathrm{~mm}$. As a precaution four 
more pictures were taken from the distance $550 \mathrm{~mm}$.

The pictures of the specimens were taken from each corner with the angle between the concrete surface and the camera lens being as small as possible, as high angles resulted in blurred images.

240 As the amount of light was limited, some of the camera settings were adjusted, see table 2 for the values.

\subsubsection{Point cloud generation}

For the generation of the poinclouds, the software EyesMap3D was used. EyesMap3D is an imaging software, where 3D models and point clouds can be generated, based on 2D images.

EyesMap3D requires calibration of the camera and objective used [21]. For the calibration of the camera and objective, 8 pictures of the concrete were used. A fully automatic orientation was chosen, where the program finds corresponding points in all pictures and estimates the location of the camera for each picture taken relative to the location of the object. The quality of the orientation is assessed based on two different values: the number of iterations and the orientation error.

The number of iterations describes the number of attempts EyesMap3D uses to pair the pictures. The optimal value is here 3 , whereas a value of 10 or more indicates problems with the orientation. The orientation error is a value between 0.5 and 2 . A 255 value between 0.5 and 1.5 is acceptable whereas a value higher than 1.5 indicates an error that is too big.

When the calibration is carried out, the point cloud can be generated. The setting for the quality of the point cloud was chosen as high.

In order to create the point cloud, EyesMap3D uses principal images. These pictures must contain everything that should be in the point cloud. The principal images were chosen automatically by EyesMap3D.

\section{Processing of data}

To create an alternative method for preparation of specimens for air void analysis, programming and computing was needed. Each of the 15 point clouds ( 3 levels of 
grinding for each of 5 concrete mixtures) contains a set of points, see section 2.2.4.

Each point is described by its x-, y-, and z-coordinate. The desired outcome was a black/white image, which could be compared with the manual contrast enhancement following the reference method [3].

The processing of the data was handled in different ways, which will be described in the following section. All programming was done in Matlab.

\subsection{General procedure}

The digital air void analysis involved the following processing steps:

- Pre-processing of the point cloud

- Identification of the surface

- Identifications of air voids (and hollows below the surface)

- Generation of a black/white image

- Digital air void analysis

\subsection{Pre-processing of the point cloud}

First the point cloud is loaded into Matlab as a ply-file. At this point, the point cloud includes everything in the photos, also the surroundings of the specimen. An approximated plane consisting of the majority of the points is generated, and all points far away from the plane are removed. This reduces the amount of distortion.

Afterwards the plane is projected into the $\mathrm{x}_{-}, \mathrm{y}$-plane to simplify the further data processing. All points not being on the concrete specimen were removed manually by creating boundaries for the point cloud, and then removing all points outside these boundaries. When the point cloud was modified to only contain points from the surface of the concrete specimen, the point cloud could be analysed. 


\subsection{Identification of the surface}

290

To analyse the air voids, the surface of the concrete specimen must be estimated.

Two different approaches of estimating the surface of the point cloud were used:

- Estimation by using the plane equation

- Surface from constant z-value - histogram

\subsubsection{Estimation by using the plane equation}

The first approach was to use the plane equation to identify the plane which corresponds to the surface of the concrete specimen. The plane equation is:

$$
a x+b y+c z+d=0
$$

295 would, in optimal conditions, result in a plane located slightly below the actual surface.

\subsubsection{Surface from constant z-value - Histogram}

The second method for estimating the concrete surface required the generation of a histogram of the z-values, and then choosing the fraction of the points which should be below the surface. Then the surface z-value could be estimated. This was an iterative procedure as the fraction had to be derived from the histograms distribution.

\subsection{Identification of the air voids (and hollows below the surface)}

Two different methods for creating a black/white image were used. Both methods required the generation of a Delauney triangulation, following the principle described in section 2.2.5.

\subsubsection{The Normal Vector Method}

The first method involved the use of normal vectors in order to estimate the air void structure. For each Delauney triangle in the triangulation, a normal vector, $n_{t}$, was created. The normal vector for the $\mathrm{x}-$, $\mathrm{y}$-plane is:

$$
n_{z}=(0,0,1)
$$


The angle between the normal vectors for the Delauney triangles and the normal vector for the $\mathrm{x}-, \mathrm{y}$-plane, will differ depending on the orientation of the triangle in the concrete structure. Theoretically, for triangles on the plane part of the concrete surface, the angle will be approximately 0 . For the triangles found in air voids, the angle will theoretically be much larger, because of the inclination, except for the bottom of the air voids, where the angle also will be approximately 0 . The principle behind the normal vector method is shown in figure 4

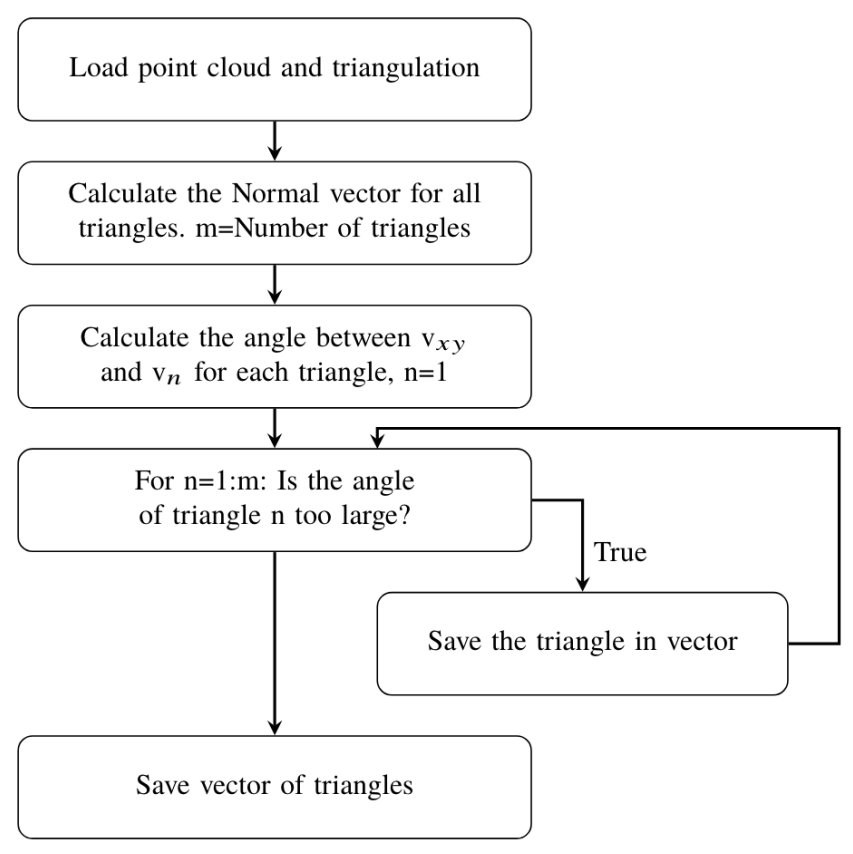

Figure 4: Algorithm of "The Normal Vector Method"

The point cloud and the triangulation are loaded, and the normal vector for each triangle is calculated. Afterwards, each triangle is analysed one at a time. If the angle between $n_{t}$ and $z$ is too high to be considered part of the surface, the triangle is considered a part of an air void. When all triangles have been analysed, a vector consisting of the triangles in air voids is saved. 


\subsubsection{Triangles Below The Plane}

The second method generates the black/white image by investigating whether each triangle is below the surface of the concrete or not. If so, it is considered part of an air void. The procedure is shown in figure 5 .

In order to determine whether a triangle is below the surface, a surface must be estimated as described in section 4.2. When the surface, the point cloud, and the triangulation are loaded, the triangles can be analysed one at a time, by looking at the three vertices in each triangle. If all three vertices have a lower z-coordinate than the estimated surface, the triangle is considered below the surface. After all triangles have been analysed, a vector consisting of the triangles is saved.

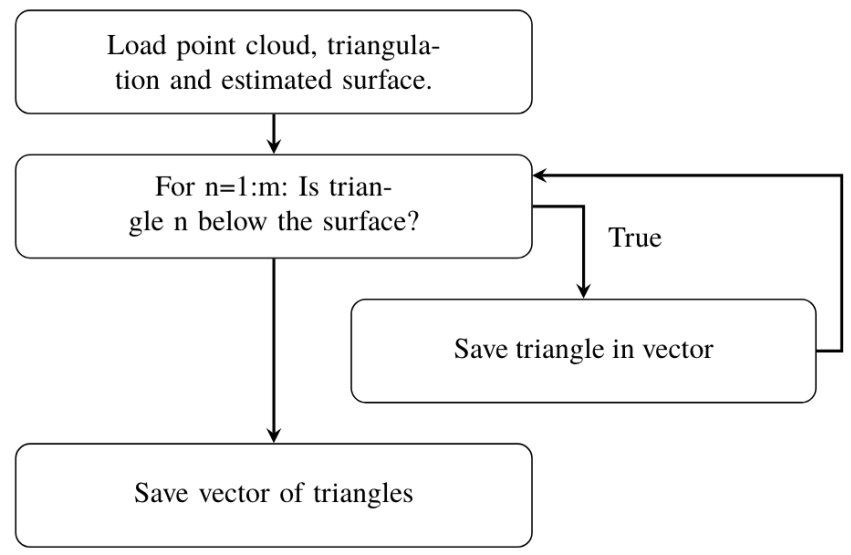

Figure 5: Algorithm of "The Triangles Below The Surface"

The deviation between the actual and the printed air void shape will decrease the smaller the triangles in the Delauney triangulation, see figure 6.

\subsection{Generation of the black/white image}

The black/white images were generated in a user-specified resolution. This was done by generating a $2 \mathrm{D}$ binary array, where the number of rows and columns corresponds to the number of locations which were analysed in the $\mathrm{x}$ and $\mathrm{y}$ direction which were 

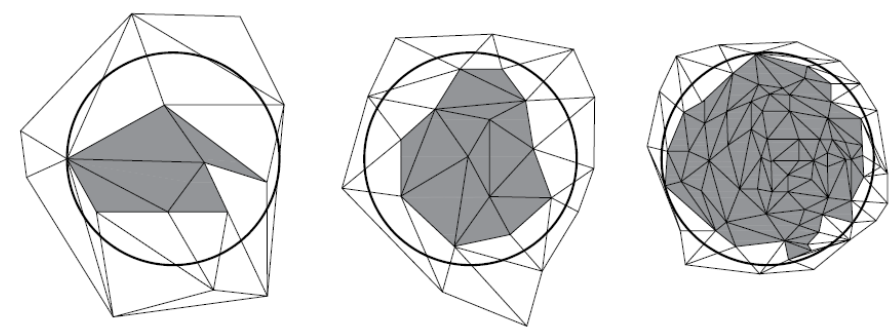

Figure 6: Difference in the shape of the pores depending on the number of triangles

analysed. The total number of points is equal to the number of pixels in the black/white image generated by this method. Every analysed location corresponds to one pixel in the generated image. If the location is within a triangle below the surface the value of the corresponding pixel is set to 1 . Otherwise, the pixel value is set to 0 . As a result, a black/white image is generated with black pixels (value 0) indicating concrete and white pixels (value 1) indicating air voids. The principle of this method is shown in figure 7 .

Load vector of triangles in the air voids

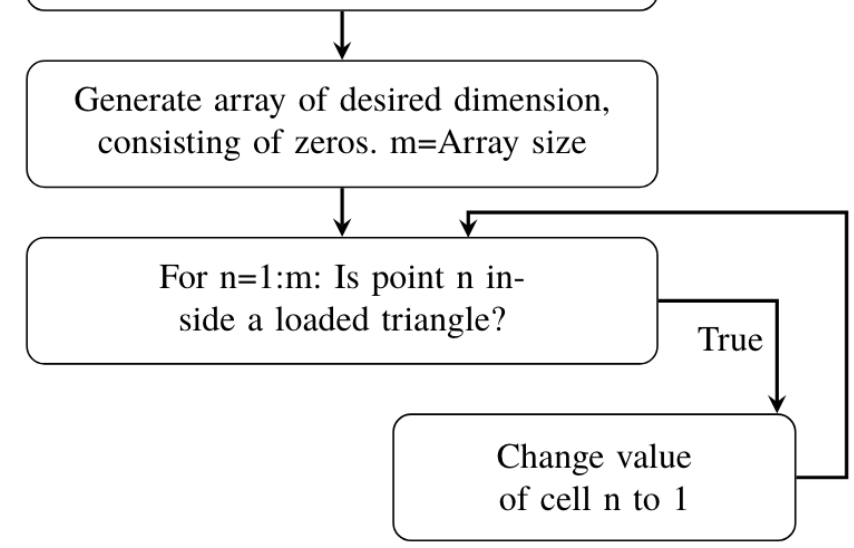

Figure 7: Generation of the black/white image 


\subsection{Digital air void analysis}

The black/white images created were analysed digitally as described in ASTM C 457 [3]. The desired traverse length determines how many traverse lines have to be distributed on the image. For each traverse line, a row is analysed, where the number of 1 's and the groups of 1's in a row is counted. The principle is shown on figure 8 and figure 9 .

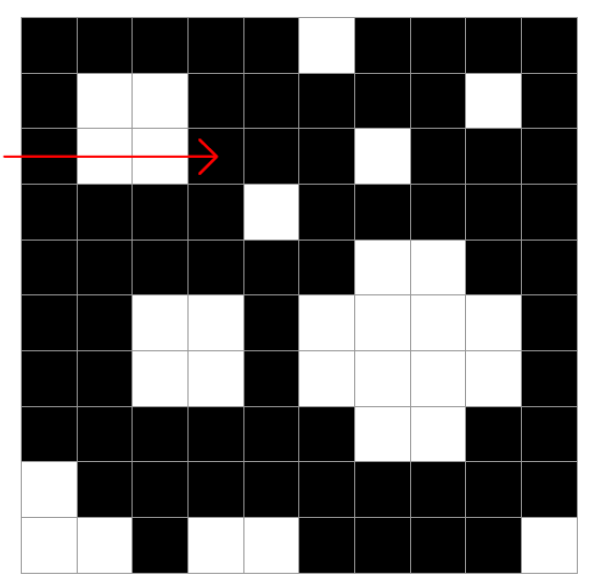

Figure 8: Analysis of pixels

From this digital analysis, the value of $\mathrm{N}, T_{t}$, and $T_{a}$ are calculated, which are then used to determine the air content, the specific surface and the spacing factor described in section 2.1

The resulting amount of air was verified, by determining the amount of air with a more simple approach.

The total projected area for all triangles below the surface and the total area percent of triangles laying below the surface were calculated. This would correspond to the amount of air in the concrete. This method is referred to as "The projected triangle area method". 


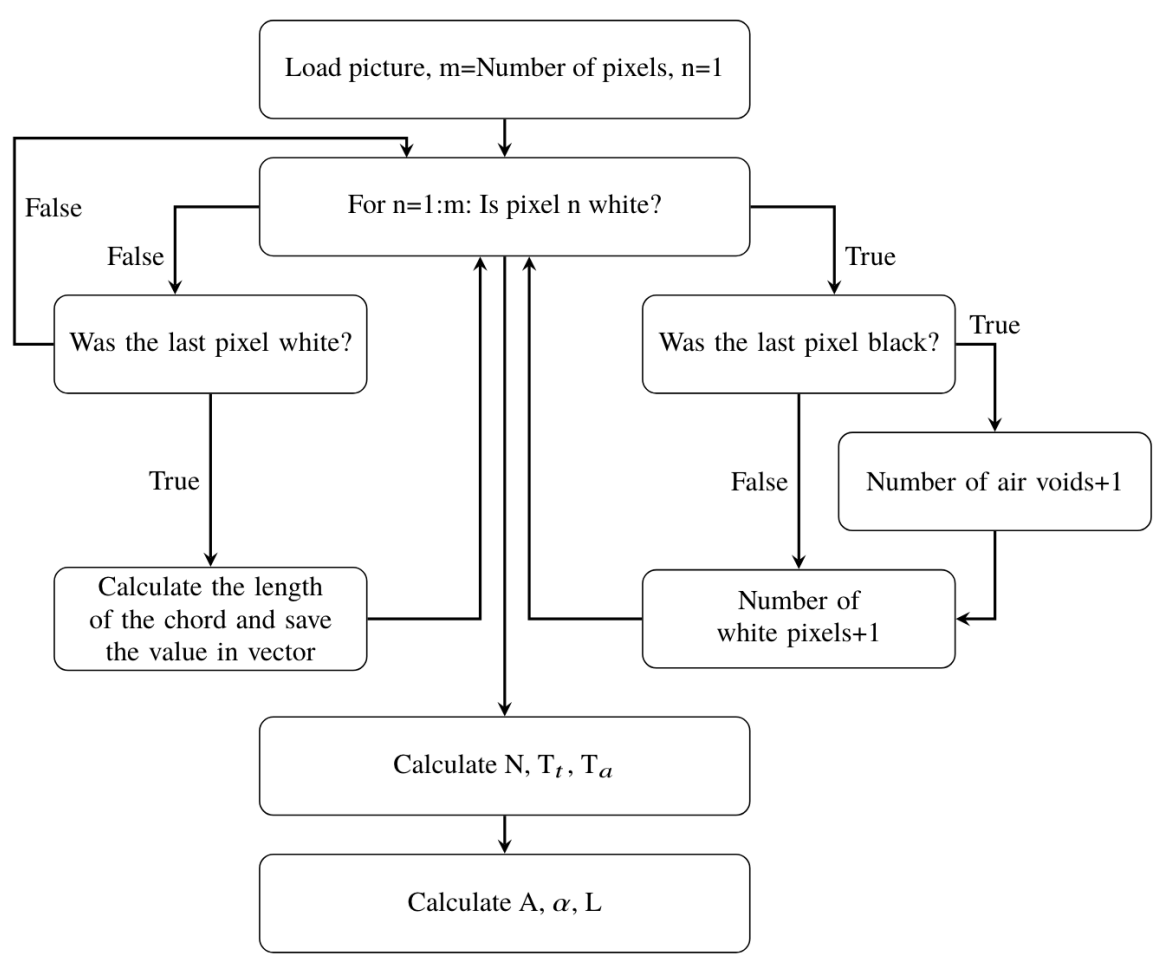

Figure 9: Principle of "The digital air void analysis"

\section{Results and discussion}

\subsection{Traditional air void analysis}

A traditional air void analysis was made for each specimen as described in section

365 3.2 and the results from this analysis are shown in table 3 together with the air content measured in the fresh concrete.

The air content was both found with and without correction of the voids in the traditional air void analysis. The air content without correction also contain the porosities in aggregates and pull-outs made during specimen preparation, while the air content with correction is where these "false" air voids are corrected and removed.

A variation from the air content in the fresh concrete and the air content in the hardened concrete is observed. The air content in the hardened concrete is considered the most reliable value, and is used as reference in the further work. The paste content 
Table 3: Air void characteristics for the 5 different specimens

\begin{tabular}{llllll}
\hline $\begin{array}{l}\text { Dosage of } \\
\text { air entrain- }\end{array}$ & $\begin{array}{l}\text { Air content } \\
\text { in fresh }\end{array}$ & $\begin{array}{l}\text { Air content } \\
\text { without }\end{array}$ & $\begin{array}{l}\text { Air content } \\
\text { with }\end{array}$ & $\begin{array}{l}\text { Specific } \\
\text { surface, }\end{array}$ & $\begin{array}{l}\text { Spacing } \\
\text { factor, }\end{array}$ \\
{$[\%$ correction } & correction & $\boldsymbol{\alpha}$ & $\overline{\boldsymbol{L}}$ \\
\hline 0.00 & 2.8 & 4.1 & 2.8 & 22 & 0.32 \\
0.05 & 2.4 & 2.5 & 2.1 & 15 & 0.55 \\
0.10 & 4.8 & 3.3 & 3.2 & 13 & 0.51 \\
0.20 & 4.1 & 3.7 & 3.5 & 18 & 0.35 \\
0.50 & 7.0 & 5.9 & 6.3 & 28 & 0.18 \\
\hline
\end{tabular}

used to calculate the spacing factor was deduced from the mix design.

375 5.2. Generation of point clouds

The number of iterations and the orientation error, described in section 3.3.3, for each grinding for each specimen is shown in table 4

It is seen that none of the values exceeds the maximum amount of iterations of 10 and the maximum orientation error of 1.5 .

${ }_{380}$ The difference in the values indicates a variation on the quality of the point clouds. It is concluded that the results not only depends on the grinding but also of the quality of the point cloud generated in EyesMap3D.

A data set comprising all photos and generated point clouds is accessible in [22].

\subsection{Generation of black/white images}

${ }_{385}$ The data were processed as described in section 4, using several methods. The result of each method, together with its strengths and weaknesses, will be described and discussed in this section.

\subsubsection{Normal vector method}

In the normal vector method, angles between $v_{x y}$ and $v_{n}$ were determined. It was expected that more than $90 \%$ of the normal vectors would be on the horizontal flat 
Table 4: Number of iterations and orientation errors.

\begin{tabular}{llll}
\hline Dosage of air entraining agent $[\% 0]$ & Grinding & No. of iterations & Orientation error \\
\hline 0.00 & 240 & 9 & 1.15 \\
0.00 & 600 & 7 & 1.10 \\
0.00 & 1000 & 7 & 1.02 \\
0.05 & 240 & $*$ & $*$ \\
0.05 & 600 & 3 & 0.66 \\
0.05 & 1000 & 10 & 1.29 \\
0.10 & 240 & 10 & 1.30 \\
0.10 & 600 & 4 & 0.73 \\
0.10 & 1000 & 4 & 0.89 \\
0.20 & 240 & 7 & 1.03 \\
0.20 & 600 & 8 & 1.09 \\
0.20 & 1000 & 10 & 1.30 \\
0.50 & 240 & 5 & 0.88 \\
0.50 & 600 & 3 & 0.65 \\
0.50 & 1000 & 10 & 1.29 \\
\hline
\end{tabular}

* The values for the specimen with $0.05 \%$ air entraining agent with grinding 240 is missing, because the values accidentally were not saved in EyesMap3D.

part of the surface (not air voids) with angles close to zero. A histogram of the angles showed that the angles were higher than expected, and therefore the black/white image for the surface could not be generated. Figure 10 shows a histogram for the angles of the specimen with $0.50 \%$ air entraining agent, ground with grain size 600 .

The high angles are caused by the roughness of the generated surface, which in turn are caused by the deviation in the $\mathrm{z}$-value for all the points in the defined concrete surface. A more rough surface would result in higher angles than a plane surface. Figure 11 illustrates the influence of the surface roughness of the point cloud.

As the normal vector method estimates the air voids from the inclination of the 


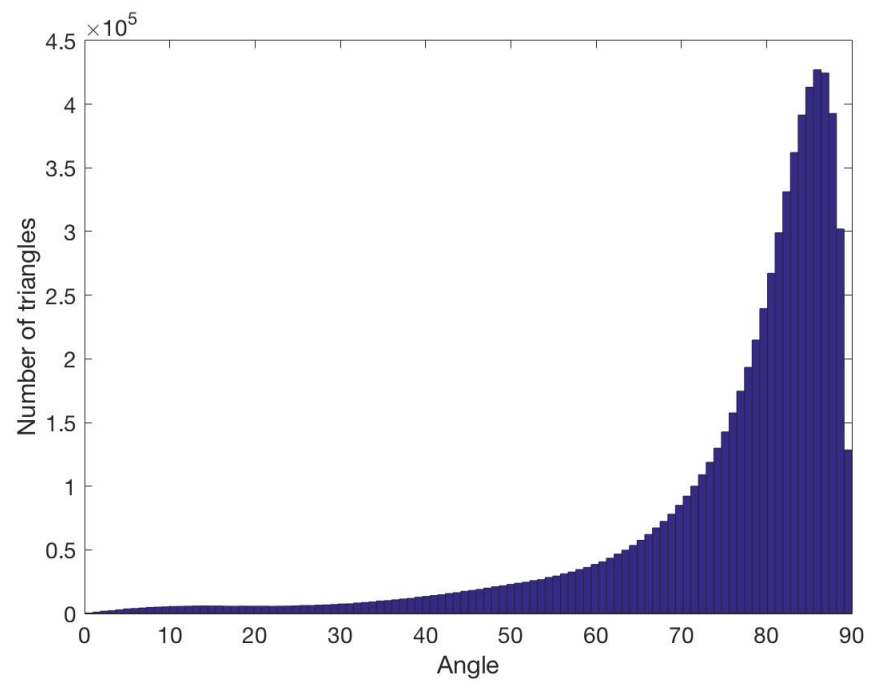

Figure 10: Histogram showing the angle between $v_{x y}$ and $v_{n}$ for the specimen with $0.50 \%$ air entraining agent ground with grain size 600 .

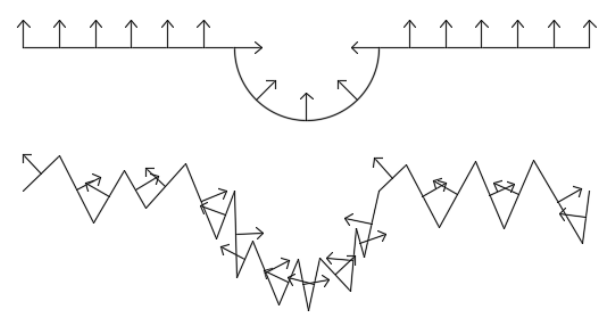

Figure 11: Influence of the surface roughness of the point cloud on direction of the normal vectors

surface, the method will identify the bottom of the air voids as concrete surface, as the angle will approximate $0^{\circ}$. In addition the method is heavily influenced by the deviation of the z-values. This deviation is caused by the noise involved in the process of generating the point cloud. As EyesMap3D produces a point cloud with a very high number of points, the horizontal distance between the points will be low, and therefore the inclination of the triangles will be higher. This will result in higher angles, see figure 11 The normal vector method is therefore deemed insufficient. 


\subsubsection{Triangles below the surface}

The air void structure was analysed using the "Triangles below the surface" method. The surface was estimated in the two different ways described in section 4.3 .

The roughness of the surface affected the use of the plane equation, as all the points located below the surface will be considered air voids. Thus the noise in the point cloud will influence the air void structure. Figure 12 shows the surface estimated in the optimal case, and with large amount of noise.

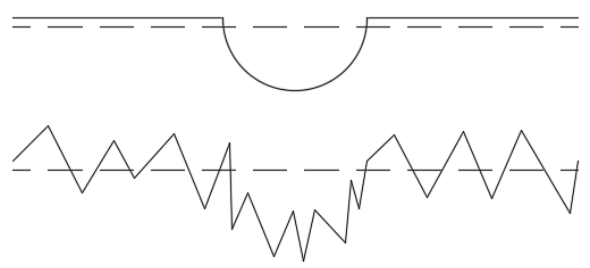

Figure 12: Estimation of the surface in the optimal case and the case with noise in the point cloud

The second method for estimating the concrete surface was to use histograms for the z-values of the point clouds. From the histograms a \%-value of the points below the surface could be estimated.

In section 5.3.1 the degree of grinding and the mean value of the angle showed no correlation. However, the histograms for the $\mathrm{z}$-values showed that the point clouds with the biggest deviation in the z-values was the specimen ground with grain size 1000, and the smallest deviation occurred with grain size 600 , see figure 13 For all point clouds, it was assumed that $8 \%$ of the points was located below the surface. The choice of a constant \%-value for all point clouds was made based on results that showed challenges with the plotting of the smallest air voids. This challenge will be discussed more in section 5.4. A black/white image for a smaller area of the specimen with $0.00 \%$ air entraining agent including an air void, is shown on figure 14 . The white pixels indicates a point below the surface, where the black pixels are part of the concrete surface. As shown, the histogram method produced the results with least noise, and the air void analysis was done using these images. In total, 15 black/white images were generated 
with this method, one for each point cloud.
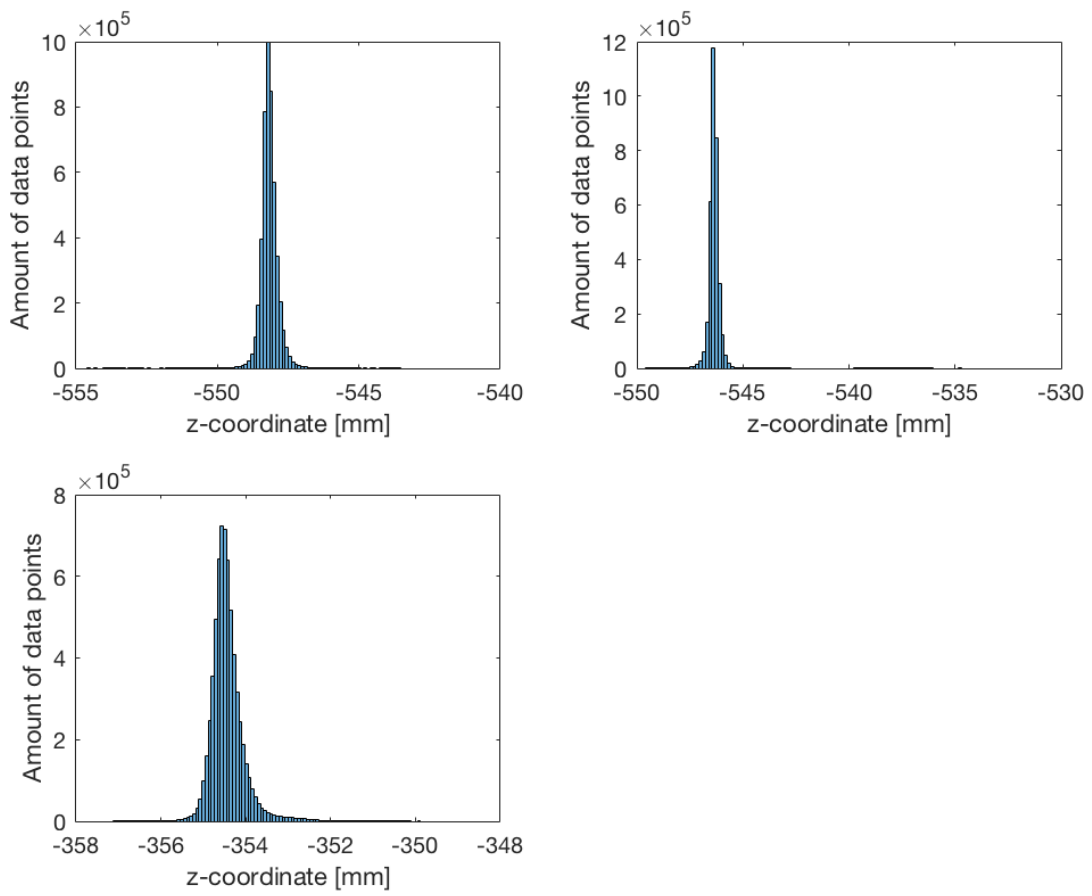

Figure 13: Histograms of $z$-values for the specimen with $0.50 \%$ air entraining agent: (a) Ground with 240 .

(b) Ground with 600. (c) Ground with 1000.

\subsection{Digital air void analysis}

The 15 black/white images have been analysed digitally, and the air void parameters have been estimated, using the digital air void analysis method, described in section 4.6 with a traverse length of $1200 \mathrm{~mm}$. Figure 15 shows the manual black/white contrast enhancement for the specimen with $0.50 \%$ air entraining agent, as well as the corresponding digital black/white image, generated from photos where the specimen was ground with grain size 600 . The images shows great visual correlations, and the larger air voids identified. As expected the digital black/white images shows that several of the smallest air voids are not generated, and "false" air voids caused by the noise also 440 appear. 


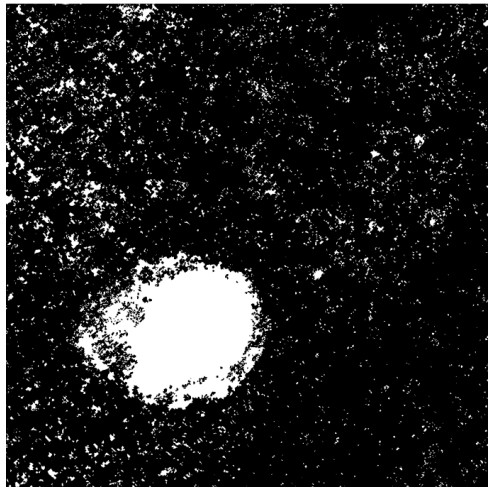

(a)

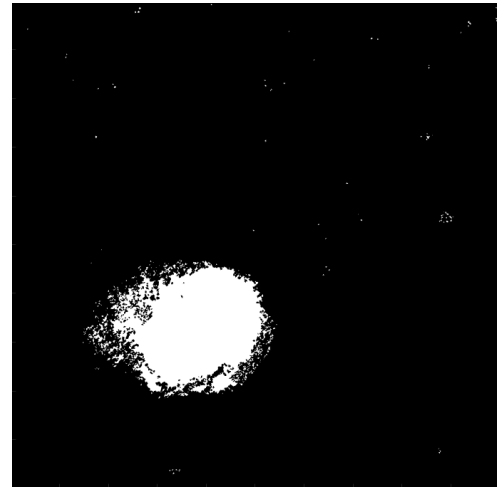

(b)

Figure 14: Black/white image of an $21 \mathrm{~mm}$ x $21 \mathrm{~mm}$ area, generated by using: (a) The plane equation. (b) Assumptions of $8 \%$ of points below the surface.

The correlation between the amount of air estimated from the manual and digital air void analysis, has been investigated. The results derived using the manual analysis was done both with and without correcting the air voids. As the digital air void analysis could not correct the air voids, the comparison would only be valid if no correction was done in the manual method. The comparison is done in figure 16 , and shows little correlation.

Figure 16 shows that the estimated amount of air using the digital air void analysis is considerably lower than the manually estimated amount. This is due to the challenges with generation of the smallest air voids in the black/white images, as well as the distortion in the point clouds. For the ideal case with no distortion and a plane surface, the percentage value chosen from the histograms will correspond to the percentage value of the air content. However, as the methods determines the air voids for the triangles where all vertices are below the surface, and not the individual points, the distortion will cause a deviation between the percentage of points beneath the surface, and the percentage of projected triangle area beneath the surface. This deviation is greatly influenced by the noise in the point clouds.

The spacing factor and specific surface were also estimated digitally for each specimen, and are shown in table 5 


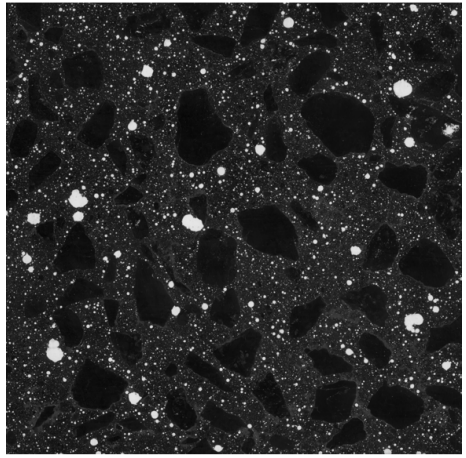

(a)

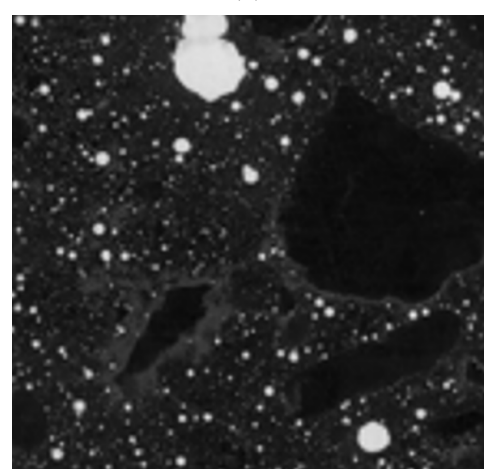

(c)

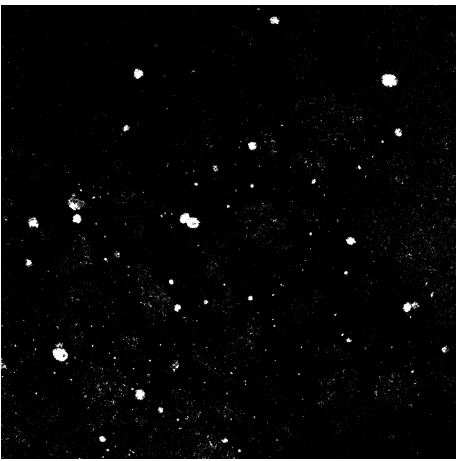

(b)

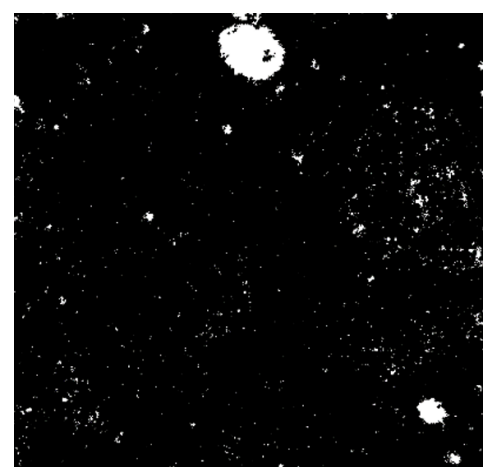

(d)

Figure 15: Specimen with $0.50 \%$ air entraining agent (6.3\% air): (a) Manual contrast enhancement with dimension $105 \mathrm{~mm}$ x $100 \mathrm{~mm}$, (b) Digital black/white image, with grain size 600 and dimension $105 \mathrm{~mm}$ x $100 \mathrm{~mm}$, (c) Bottom left corner of manual contrast enhancement with dimention $25 \mathrm{~mm}$ x $25 \mathrm{~mm}$, (d)

Bottom left corner of digital black/white image, with grain size 600 and dimension $25 \mathrm{~mm}$ x $25 \mathrm{~mm}$.

Even though the method showed problems with generating the smallest air voids, the specific surface was estimated higher and the spacing factor was estimated lower for nearly all the black/white images using the digital method. This was due to distortion in the point clouds, which generated a high amount of "false" air voids, many with the size corresponding to a single pixel in the black/white image. The distortion also caused some of the larger air voids to be plotted as a high number of small air voids, see figure 14 Therefore, a proper comparison between the spacing factor and specific surface estimated with each method could not be done.

The digitally estimated amount of air was checked, as described in section 4.6 . 


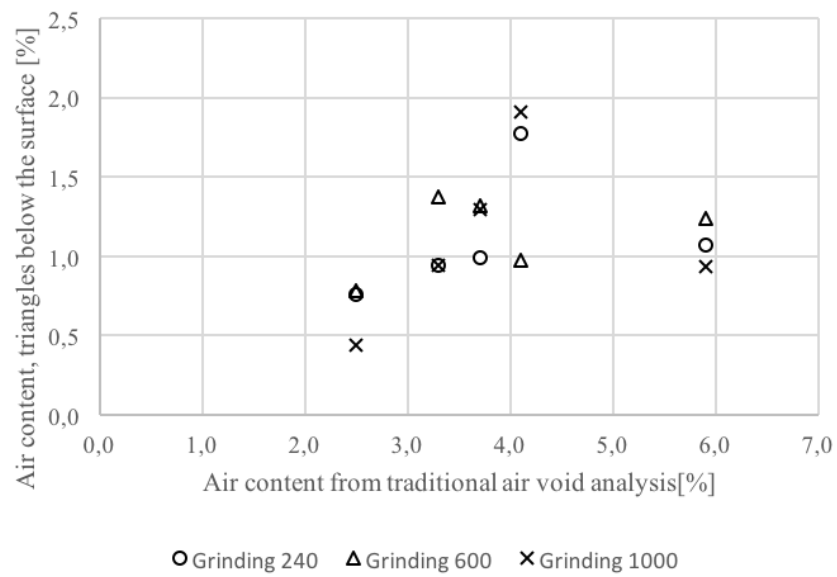

Figure 16: Relation between the amount of air estimated from the digitally black/white images and the traditional air void analysis

Table 5: Specific surface and spacing factor for the black/white images generated from the specimens ground ved grain size 600 , and the results from the traditional air void analysis

\begin{tabular}{|c|c|c|c|c|}
\hline $\begin{array}{l}\text { Amount of air } \\
\text { entraining agent }[\% 0]\end{array}$ & $\begin{array}{l}\text { Digital, } \\
\alpha\left[\mathrm{mm}^{-1}\right]\end{array}$ & $\begin{array}{l}\text { Digital, } \\
\bar{L}[\mathrm{~mm}]\end{array}$ & $\begin{array}{l}\text { Traditional, } \\
\alpha\left[\mathrm{mm}^{-1}\right]\end{array}$ & $\begin{array}{l}\text { Traditional, } \\
\bar{L}[\mathrm{~mm}]\end{array}$ \\
\hline 0.00 & 40.85 & 0.27 & 12.00 & 0.51 \\
\hline 0.05 & 59.30 & 0.21 & 16.00 & 0.47 \\
\hline 0.10 & 36.03 & 0.27 & 15.00 & 0.46 \\
\hline 0.20 & 36.64 & 0.27 & 18.00 & 0.36 \\
\hline 0.50 & 47.88 & 0.21 & 25.00 & 0.20 \\
\hline
\end{tabular}

Figure 17 shows the relation between the amount of air estimated using the digital air void analysis, and the amount of air estimated by calculating the percentage of the 470 projected triangle area below the surface. The correlation shows that the digital air void analysis produces reasonable results, given the black/white images. 


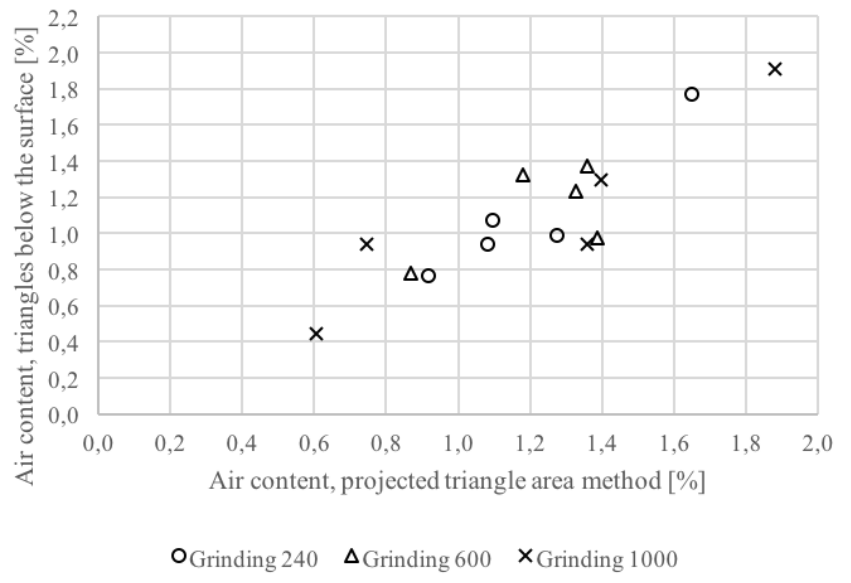

Figure 17: Relation between the amount of air estimated from the digitally air void analysis and "The projected triangle area method"

\subsection{Suggestions for improvement of the method}

The proposed method rely on both high quality photos and data processing performed by commercial software.

\subsubsection{Alternative choice of camera}

With the proposed method, it is not possible to detect air voids smaller than the MOS of the camera, which for the Nikon camera sets a limit of $17.85 \mu \mathrm{m}$. When generating the black/white images, chord lengths considered part of air voids shorter than the MOS were observed. However, these may be considered artefacts of the method. When generating the point cloud, some points when projected into the $\mathrm{x}, \mathrm{y}$-plane may be closer to each other than MOS, and due to scatter, they may be classified as air voids.

The detection of small air voids is important for the outcome of the air void analysis. Therefore, one way to improve the photogrammetric method is to use a camera with smaller MOS. In this project, actually the first choice of camera was the camera in a Nokia Lumia 1020 cell phone. The specifications for this camera are shown in table 6 .

The Nokia camera has a smaller pixel size than the Nikon camera, and together with the combination of focal length and focus distance, the MOS is only $6.46 \mu \mathrm{m}$, i.e. approximately one third of the MOS of the Nikon camera. 
Table 6: Specifications for the Nikon camera and the Nokia Lumia 1020

\begin{tabular}{lll}
\hline Technical specifications & Nikon camera & Nokia Lumia 1020 \\
\hline Resolution & 24.3 Megapixel & 41.0 Megapixel \\
Focal length & $105 \mathrm{~mm}$ & $26 \mathrm{~mm}$ \\
Pixel size & $5.97 \mu \mathrm{m}$ & $1.12 \mu \mathrm{m}$ \\
Focus distance & $314 \mathrm{~mm}$ & $150 \mathrm{~mm}$ \\
MOS & $17.85 \mu \mathrm{m}$ & $6.46 \mu \mathrm{m}$ \\
\hline
\end{tabular}

However, the Nokia camera turned out not to be compatible with the Eyesmap3D software. EyesMap3D works with Exif meta data, and the meta data of the photos taken by the Nokia camera was in the Exiv2 format. This made it impossible to load the photos into EyesMap3D. Moreover, it was foreseen that the smaller MOS of the Nokia camera was more on the paper than in reality. Only the part of the concrete specimen closest to the camera would be $150 \mathrm{~mm}$ from the lens, whereas for the part of the specimen furthest away, the distance to the lens would be approximately twice as long, so here the MOS is also larger. This effect is more pronounced for the Nokia camera than for the Nikon camera, because the Nokia camera shoots the photos with a wider angle.

\subsubsection{Alternative choice of software}

In the search of an alternative to EyesMap3D the software ReCap Photo from Autodesk was tested [23]. This software seemed more user-friendly since no calibration nor orientation was needed. To compare the two software packages, the same photos were loaded into both software packages (using the specimen with $0.50 \%$ air entraining agent with grinding 600 as an example).

- ReCap Photo generated a point cloud with 948,058 points

- EyesMap3D generated a point cloud with 8,934,063 points

Thus, Eyesmap3D generated 9.42 times as many points as ReCap Photo, meaning that the mean distance between the points in the $\mathrm{x}, \mathrm{y}$-plane is increased $\sqrt{9.42}=3.07$ 
times, when switching from EyesMap3D to Recap Photo. This corresponds to a drastic

loss of resolution.

Qualitatively, it looked like the z-coordinate for each point was less scattered, when using ReCap Photo compared to EyesMap3D. However, this probably also could have been obtained by applying a smoothening algorithm to the point cloud generated by EyesMap3D if allowing for a reduction of points. If a choice has to be made between relying on the software, which virtually is a black box.

\subsubsection{Final evaluation}

The aim of the project was to establish an automated procedure based on photogrammetry, which enables air void analysis of concrete plane sections without previous contrast enhancement. This has been done by essentially putting three building blocks together:

- A camera. The camera has to be chosen wisely based on camera specifications, especially emphasising the MOS, but it need not be an expensive camera; it could even be a cell phone camera.

- A commercial software package that can translate digital photos into a point cloud (photogrammetric analysis)

- Matlab procedures specially made for the project; Matlab procedures have been used to separate the test surface from the surroundings in the point cloud, to identify air voids on the test surface, and to perform the air void analysis based on chord counting in a digital image.

In round figures, the resolution of the method is $20 \mu \mathrm{m}$. This cannot compete with methods, where the plane section is examined under the microscope. Ideally, there should be more than one pixel in each air void, to allow for some filtering, so to identify air voids down to $10 \mu \mathrm{m}$, a resolution of at least $5 \mu \mathrm{m}$ is needed. It would be possible to fine-tune the method by changing lighting and camera settings, etc. However, for the time being, a resolution of e.g. $5 \mu \mathrm{m}$ is out of reach. 
It all links to the MOS of the camera. A small MOS can even compensate for a less optimum choice of software for photogrammetric analysis, because if the digital input photos are very detailed, it is acceptable if the resolution of the generated point cloud is less than the resolution of the photos. Within computer science, there is a famous law known as Moore's law, which predicts that the chip performance and therefore the computational power of personal computers doubles every 18 months. There is a similar law for image processors. This law, Hendy's law, predicts that the number of pixels that can be purchased for one dollar increases by a factor of 10 every 5 years [24], i.e. the resolution increases by a factor of $10 / \sqrt{2}=7$ every 5 years. Projecting Hendy's law into the future, it means that the resolution of the method proposed in this project may be as good as $3 \mu \mathrm{m}$ in 5 years time due to the general improvement of digital camera technology.

\section{Conclusion}

In the presented project, a new method for air void analysis of hardened concrete based on photogrammetry is tested. The new method eliminates the need of contrast enhancement, which is often used in standardised methods such as ASTM C457 (reference method of the project). When using the new method, one of the outputs is a digital image, where air voids are shown as white areas and the rest of the image is black. The digital black/white image can be compared to the test surface of the concrete specimen after traditional contrast enhancement. Visually, a clear correlation was found between the digital black/white image and the test surface after manual contrast enhancement.

However, when comparing quantitative measures such as total air content, specific surface of air voids, and spacing factor, rather large differences are observed between results obtained by the new and the traditional method. This is due to

- the resolution of the new method. The resolution is approx. $20 \mu \mathrm{m}$, and this is not as good as the reference method. Therefore, many of the smaller air voids in air entrained concrete are not identified by the new method

- scatter in the digital elevation model of the new method, which result in generation of "false" air voids in the black/white image 
The imperfections of the output in the form of the black/white image are caused by limitations of the camera and the uncertainties of generating the point clouds (here, the software EyesMap3D was used). Because of the development of both cameras and software packages, the new method may prove useful in the future, but as for now, the 570 method is not applicable because of the above-mentioned uncertainties.

\section{Acknowledgements}

Plane sections were prepared by PELCON A/S, and it was also laboratory staff at PELCON A/S, who conducted the standardised air void analysis that were used as references in the project. This help as well as valuable discussions with PELCON A/S director Peter Laugesen regarding how to obtain the best quality air void analysis are greatly acknowledged.

\section{References}

[1] F. H. Jackson, et al., Concretes containing air-entraining agents, ACI Journal Proceedings 40 (1944) 509-569.

[2] T. C. Powers, The air requirement of frost resistant concrete, ACI Journal ProceedingsProceedings of the Highway Research Board 29 (1949) 184-221.

[3] A. international, C 457-16: Standard Test Method for Microscopical Determination of Parameters of the Air-Void System in Hardened Concrete, ASTM international (2016).

[4] K. W. Peterson, Automated air-void system characterization of hardened concrete: Helping computers to count air-voids like people count air-voids - methods for flatbed scanner calibration, Ph.D. thesis, Michigan Technological University (2008).

[5] A. H. Sommer, The precision of the microscopical determination of the air-void system in hardened concrete, Cement, Concrete, and Aggregates 1 (2) (1979) $49-55$. 
[6] B. B. W. Langan, M. A. Ward, Determination of the air-void system parameters in hardened concrete âĂŞ an error analysis, ACI Journal 83 (6) (1986) 943-952.

[7] R. G. C. Richard Pleau, Patrick Plante, M. Pigeon, Practical considerations per-

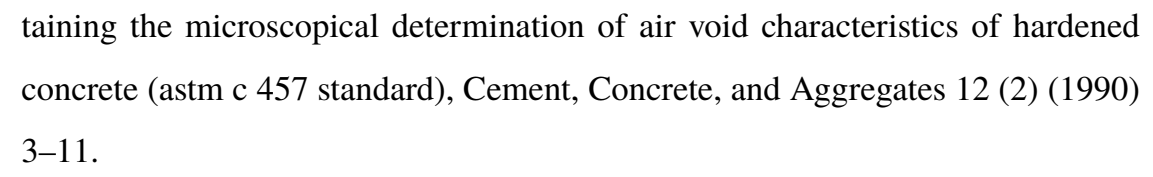
taining the microscopical determination of air void characteristics of hardened concrete (astm c 457 standard), Cement, Concrete, and Aggregates 12 (2) (1990) $3-11$.

[8] R. P. D. Francois Saucier, D. Vezina, Precision of the air void characteristics measurements by astm c 457: Results of an interlaboratory test program, Can. J. Civ. Eng. 23 (5) (1996) 1118-1128.

[9] S. Chatterji, H. Gudmundsson, Characterization of entrained air bubble systems in concrete by means of an image analyzing microscope, Cement and Concrete Research 7 (1977) 423-428.

[10] U. H. Jakobsen, et al., Automated air void analysis of hardened concrete - a round robin study, Cement and Concrete Research 36 (2006) 1444-1452.

[11] D. standard, DS/EN 480-11: Admixtures for concrete, mortar and grout - Test mathods - Part 11: Determination of air void characteristics in hardened concrete, Danish standard (1998).

[12] E. J. Elsen, Automated air void analysis on hardened concrete. results of a european intercomparison testing program, Cement and Concrete Research 31 (7) (2001) $1027-1031$.

[13] J. E. L. F. BjÃürn Schouenborg, M. SandstrÃüm, Air and air void structures in concrete âĂŞ general overview and picture atlasm, Nordtest project 1121-93, SP report 1995:51, Swedish National Testing and Research Institute.

[14] M. T. Hasholt, et al., Frost resistance of concrete with high contents of fly ash a study on how hollow fly ash particles distort the air void analysis, Cement and Concrete Research 119 (2019) 102-112. 
[15] R. Pleau, et al., Some findings on the usefulness of image analysis for determining the characteristics of the air-void system on hardened concrete, Cement, Concrete and Composites 23 (2-3) (2001) 237-246.

[16] J. Baerentzen, et al., Guide to computational geometry processing. Foundations, algorithms, and methods, Springer, 2012.

[17] Y. Song, et al., Advances in measuring air-void parameters in hardened concrete using a flatbed scanner, Journal of Testing and Evaluation 45 (5) (2017) 17131725.

[18] P. K. Mehta, P. J. M. Monteiro, Concrete: Microstructure, Properties, and Materials, 4th Edition, McGraww-Hill Education, 2014.

[19] E. M. Mikhail, et al., Introduction to Modern Photogrammetry, John Wiley and Sons, 2001.

[20] K. Atkinson, Close Range Photogrammetry and Machine Vision, Whittles Publishing, 1996.

[21] eCapture, eyesMap3D software - user manual, eCapture (2016).

[22] F. A. H. Uhre, et al., Air void analysis of hardened concrete by means of photogrammetry (dataset) (2019). URL https://doi.org/10.11583/DTU.8191502

[23] Autodesk, ReCap - Create accurate 3D models with reality capture (2018).

[24] R. A. Athale, et al., Scaling analysis of computational imaging systems, SPIE Defence and Security Symposium. 